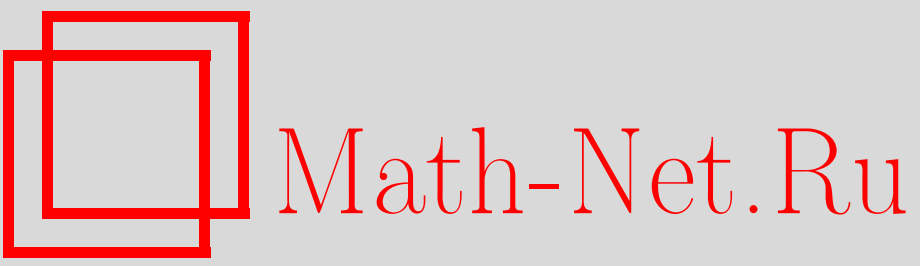

Ю. Ю. Кочетков, Гомологии нильпотентных подалгебр супералгебры Ли $K(1,1)$. II, УMH, 2000, том 55, выпуск 6, 143-144

DOI: https://doi.org/10.4213/rm346

Использование Общероссийского математического портала Math-Net.Ru подразумевает, что вы прочитали и согласны с пользовательским соглашением

http://www. mathnet.ru/rus/agreement

Параметры загрузки:

IP : 54.162 .27 .143

26 апреля 2023 г., 17:51:54 


\title{
ГОМОЛОГИИ НИЛЬПОТЕНТНЫХ ПОДАЛГЕБР СУПЕРАЛГЕБРЫ ЛИ $K(1,1)$. II
}

\author{
Ю. Ю. КОчЕтКОв
}

Обозначим $W_{n}$ алгебру Ли полиномиальных векторных полей от $n$ переменных $x_{1}, \ldots, x_{n}$. $W_{n}$ - градуированная алгебра: $\operatorname{deg}\left(x_{1}^{k_{1}} \cdots x_{n}^{k_{n}} \frac{\partial}{\partial x_{i}}\right)=k_{1}+\cdots+k_{n}-1$. Таким образом введенная градуировка позволяет градуировать цепи, циклы и гомологии. Далее мы будем, как правило, рассматривать однородные (относительно градуировки) элементы, цепи и циклы. Группы цепей, циклов и гомологий степени $m$ будут обозначаться $C_{*}^{(m)}, Z_{*}^{(m)}$ и $H_{*}^{(m)}$, соответственно.

Через $L_{k}\left(W_{n}\right) \subset W_{n}, k=1,2, \ldots$, мы обозначим нилппотентную подалгебру - линейную оболочку однородных элементов степени $\geqslant k$. Теорема Гончаровой [1] дает комбинаторные правила вычисления размерностей групп гомологий $H_{p}^{(m)}\left(L_{k}\left(W_{1}\right)\right)$ с тривиальными коэффициентами.

ТеОрема 1 (Л.В. Гончарова [1]). $\operatorname{dim}\left(H_{p}^{(m)}\left(L_{k}\left(W_{1}\right)\right)\right)=$ числу строго возрастающих последовательностей $a_{1}, \ldots, a_{p}$ натуральных чисел таких, что

1) $a_{1}+\cdots+a_{p}=m$

2) $a_{i}-a_{i-1} \geqslant 3, i=2, \ldots, p$

3) $k \leqslant a_{1}, a_{p} \leqslant 2 k+3(p-1)$

4) если $a_{1}=k$, то $a_{p}<2 k+3(p-1)$.

В работе $\Phi$. Вайнштейна [2] прояснены причины появления таких формул и дано достаточно прозрачное доказательство теоремы, используюшее серию комбинаторных тождеств, открытых Вайнштейном, а также топологические соображения.

Распространить эту технику на случай $n>1$ не удается. В данной работе предпринята попытка найти комбинаторные формулы для размерностей групп гомологий в "полутораразмерном" случае. Точнее, мы рассматриваем супералгебру Ли $W(1,1)$ полиномиальных векторных полей от одной четной и одной нечетной переменной. Эта супералгебра изоморфна супералгебре $K(1,1)$ полиномов от одной четной переменной $t$ и одной нечетной переменной $\xi$. Градуировка вводится следуюшим образом: $\operatorname{deg}\left(t^{k}\right)=2 k-2, \operatorname{deg}\left(t^{k} \xi\right)=2 k-1$, т.е. четные мономы имеют четную степень, а нечетные - нечетную. Далее моном степени $s$ будет обозначаться $e_{s}$. Скобка определяется так:

$$
\begin{aligned}
& {\left[e_{2 k}, e_{2 l}\right]=(2 l-2 k) e_{2 k+2 l},} \\
& {\left[e_{2 k+1}, e_{2 l+1}\right]=e_{2 k+2 l+2},} \\
& {\left[e_{2 k}, e_{2 l+1}\right]=(2 l+1-k) e_{2 k+2 l+1} .}
\end{aligned}
$$

Нас будут интересовать нилппотентные подалгебры $L_{k} \subset K(1,1), k=1,2, \ldots$ :

$$
L_{k}=\left\langle e_{k}, e_{k+1}, \ldots\right\rangle .
$$

Гомологии алгебры $L_{1}$ рассмотрены в [3], где сформулировано без доказательства следуюшее утверждение: $\operatorname{dim}\left(H_{p}\left(L_{1}\right)\right)=2$, причем

$$
\operatorname{dim}\left(H_{p}^{(m)}\left(L_{1}\right)\right)= \begin{cases}1, & \text { если } m=2 p^{2} \pm p, \\ 0 & \text { в остальных случаях. }\end{cases}
$$

Рассмотрим 2-гомологии алгебр $L_{k}$.

Teopema 2. $\operatorname{dim}\left(H_{2}\left(L_{k}\right)\right)<\infty$.

ДокАЗАТЕЛЬСтво. Достаточно доказать, что $H_{2}^{(m)}\left(L_{k}\right)=0$, при $m$ достаточно большом. Доказательство состоит в предъявлении набора 3-цепей таких, что их дифференциалы линейно независимы, а количество этих цепей равно $\operatorname{dim}\left(Z_{2}^{(m)}\left(L_{k}\right)\right)=\operatorname{dim}\left(C_{2}^{(m)}\left(L_{k}\right)-1\right)$ при $m>6 k$.

Работа выполнена при поддержке Российского фонда фундаментальных исследований (грант № 98-01-00329). 
Как указывалось выше, наша цель - найти формулы для размерностей групп гомологий, аналогичные формулам Гончаровой. К сожалению, найденные формулы работают только в случае гомологий 2-го порядка и дают оценку гомологиям 3-го порядка.

Рассмотрим сначала гомологии 2-го порядка. Отметим, что пространство циклов $Z_{2}^{(m)}\left(L_{k}\right)$ имеет размерность на единицу меньшую, чем пространство цепей $C_{2}^{(m)}\left(L_{k}\right)$. Базис $C_{2}^{(m)}\left(L_{k}\right)$ образуют цепи $e_{k} \wedge e_{m-k}, e_{k+1} \wedge e_{m-k-1}, \ldots$ Последним элементом базиса является цепь $e_{l} \wedge e_{l+1}$, если $m=2 l+1$, цепь $e_{2 l+1} \wedge e_{2 l+1}$, если $m=4 l+2$, и цепь $e_{2 l-1} \wedge e_{2 l+1}$, если $m=4 l$. Таким образом, размерность $Z_{2}^{(m)}\left(L_{k}\right)$ равна числу 2-последовательностей $a_{1}, a_{2}$ натуральных чисел, где $k \leqslant a_{1}<a_{2}, a_{1}+a_{2}=m$ и $a_{2}-a_{1} \geqslant 2$, если $a_{1}$ и $a_{2}$ оба четны, и $a_{2}-a_{1} \geqslant 3$, если хотя бы одно из этих чисел нечетно. Дадим определение.

ОПРЕДЕЛЕнИЕ 1 . Последовательность $a_{1}, \ldots, a_{l}$ натуральных чисел называется $(k, m)$ стандартной, если $k \leqslant a_{1}<\cdots<a_{l}, a_{1}+\cdots+a_{l}=m$ и для всех $i=1, \ldots, l-1$ выполнено следующее условие: $a_{i+1}-a_{i} \geqslant 2$, если $a_{i}$ и $a_{i+1}$ оба четные, и $a_{i+1}-a_{i} \geqslant 3$, если хоть одно из этих чисел нечетно.

Теперь мы можем сформулировать гипотезу о размерностях групп 2-гомологий.

Правило 1. Пусть $k \geqslant 3$ нечетно, тогда $\operatorname{dim}\left(H_{2}^{(m)}\left(L_{k}\right)\right)=s+t_{1}+t_{2}$, где $s-$ число $(k, m)$-стандартных 2 -последовательностей $a_{1}, a_{2}$ таких, что $a_{2} \leqslant 2 k+1$ и $a_{2} \leqslant 2 k-1$, если $a_{1}=k, a$

$$
\begin{aligned}
& t_{1}=\left\{\begin{array}{l}
1 \quad \text { при } 4 k \leqslant m \leqslant 4 k+2, \\
0 \quad \text { в остальных случаях; }
\end{array}\right. \\
& t_{2}= \begin{cases}-1 & \text { при } 3 k+8 \leqslant m \leqslant 4 k+2, \\
0 & \text { в остальньх случаях. }\end{cases}
\end{aligned}
$$

Правило 2. Пусть $k \geqslant 2$ четно, тогда $\operatorname{dim}\left(H_{2}^{(m)}\left(L_{k}\right)\right)=s+t$, где $s-$ число $(k, m)$ стандартных 2-последовательностей $a_{1}, a_{2}$ таких, что $a_{2} \leqslant 2 k+1, a$

$$
t= \begin{cases}1 & \text { при } m=3 k+4,3 k+5, \\ 0 & \text { в остальньх случаях }\end{cases}
$$

ЗАмечАние 1 . При $2 \leqslant k \leqslant 22$ справедливость гипотезы была проверена вычислениями.

Найдены аналогичные формулы для размерностей 3-гомологий, правда здесь удается получить лишь оценку размерности сверху. Как и ранее, мы отдельно рассмотрим случаи нечетного и четного $k$.

Правило 3. Пусть $k \geqslant 15$ нечетно, тогда $\operatorname{dim}\left(H_{3}^{(m)}\left(L_{k}\right)\right) \leqslant s-1$, где $s$ - әто число $(k, m)$-стандартных 3 -последовательностей $a_{1}, a_{2}, a_{3}$ таких, что $a_{3} \leqslant 2 k+3 ; a_{3} \leqslant$ $2 k+1$, если $a_{1}=k ;$ и $a_{3} \leqslant 2 k+2$, если $a_{1} \geqslant k+7$. При этом неравенство является равенством, если $m \leqslant 4 k+14$.

ПравИЛО 4. Пусть $k \geqslant 16$ четно, тогда $\operatorname{dim}\left(H_{3}^{(m)}\left(L_{k}\right)\right) \leqslant s-1$, где $s-$ это число $(k, m)$-стандартных 3-последовательностей $a_{1}, a_{2}, a_{3}$ таких, что $a_{3} \leqslant 2 k+4 u a_{3} \leqslant$ $2 k+3$, если $a_{1} \neq k+2$ или $k+3$. При этом неравенство является равенством, если $m \leqslant 4 k+11$.

ЗАмЕчАниЕ 2 . При $15 \leqslant k \leqslant 32$ справедливость гипотезы была проверена вычислениями.

\section{СПИСОК ЛИТЕРАТУРЫ}

[1] Гончарова Л.В.// Функц. анализ и его прил. 1973. Т. 7. №2. С. 6-14. [2] Weinstein F. V. // Adv. Soviet Math. 1993. V. 17. Р. 155-216. [3] Ретах В. С., Фейгин Б. Л. // УMH. 1982. T. 37. № 2. C. 233-234. 